MPP-2009-16

\title{
DOC: A code to calculate scalar one-loop four-point integrals with complex masses
}

\author{
DAO Thi Nhung and LE Duc Ninh \\ Max-planck-Institut für Physik (Werner-Heisenberg-Institut), \\ D-80805 München, Germany
}

\begin{abstract}
We present a new Fortran code to calculate the scalar one-loop four-point integral with complex internal masses, based on the method of 't Hooft and Veltman. The code is applicable when the external momenta fulfill a certain physical condition. In particular it holds if one of the external momenta or a sum of them is timelike or lightlike and therefore covers all physical processes at colliders. All the special cases related to massless external particles are treated separately. Some technical issues related to numerical evaluation and Landau singularities are discussed.
\end{abstract}




\section{Introduction}

In order to calculate the radiative corrections to reactions with unstable internal particles (like the top quark or $\mathrm{W} / \mathrm{Z}$ gauge bosons) which can be on-shell, one has to resum the propagator of these particles. This can lead to prescriptions where the squared mass of unstable particles are regarded as complex parameters with a finite imaginary part proportional to the width. It means that we have to calculate Feynman integrals with complex masses.

From the mathematical viewpoint, the introduction of a width helps to protect the cross section from several singularities, called Landau singularities [1, 2, 3, 4, 5, 6]. Let us explain this in a simple way. In order to define a Feynman integral in the case of real masses, one needs the $i \epsilon$-prescription to deform the integration contour from various poles. The final result is obtained by taking the limit $\epsilon \rightarrow 0$. We observe that the position of the poles, in general, depend on the values of internal masses and external momenta (see Appendix $\mathrm{A}$ for more details). It is therefore possible that these poles can pinch the integration contour in the limit $\epsilon \rightarrow 0$, leading to a Landau singularity. By making the masses complex, the poles are moved away from the real axis and hence all the various Landau singularities no longer exist.

The method to calculate scalar one-loop integrals with real/complex masses was explained by 't Hooft and Veltman [7]. Following this, van Oldenborgh has written a Fortran package FF to calculate one-loop integrals [8, 9]. Based on this package, a more convenient library called LoopTools which can calculate scalar and tensor one-loop integrals up to five-point has been developed by Hahn and colleagues [10, 11]. LoopTools/FF can handle one-loop integrals with complex masses up to three-point. The only missing piece in the scalar integral sector is the one-loop four-point function with complex masses. A Fortran 77 code to calculate this function is presented in this paper. The method is based on the one given in [7] where a general result is written down, see Eq. (6.26) of [7]. This result is applicable as long as the external momenta fulfill a certain physical condition. In particular it holds if one of the external momenta or a sum of them is timelike $*$. If there is at least one lightlike external momentum then similar results can be derived by using the same method. If the condition is not met then the calculation becomes mathematically quite tricky. In principle, the result in this case should be an analytical continuation of Eq. (6.26) of [7] with some extra logarithms. However, it is not easy to find these terms in practice. Fortunately, this special case is never encountered in collider scatterings where there always exists at least two timelike/lightlike external momenta.

The outline of this paper is as follows. In the next section, we give all the relevant formulae which have been implemented in our code, named DOC. All the important facts related to the code are given in Section 3. The appendices include a discussion on threepoint and four-point Landau singularities and the calculation of a basic integral.

\footnotetext{
*In our metric timelike $p$ implies positive $p^{2}$.
} 


\section{The method}

The notation used in this paper follows closely the one of Ref. [7]. The definition of logarithm, Spence function and $\eta$-function are the same. The signature of our space-time metric is $(+,-,-,-)$ which is different from the one used by 't Hooft and Veltman, however.

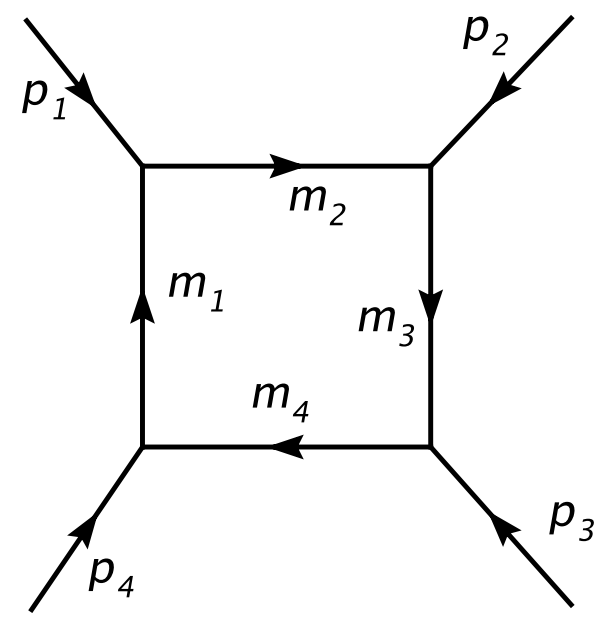

Figure 1: The box diagram.

With the momenta assigned as in Fig. 1, the scalar one-loop four-point function in 4-dimension space time is defined by

$$
D_{0}=\int \frac{d^{4} q}{i \pi^{2}} \frac{1}{\prod_{j=1}^{4}\left[\left(q+k_{j}\right)^{2}-m_{j}^{2}+i \epsilon\right]}
$$

where the momenta $k_{i}$ are related to the real external momenta as

$$
k_{1}=0, \quad k_{2}=p_{1}, \quad k_{3}=p_{1}+p_{2}, \quad k_{4}=p_{1}+p_{2}+p_{3}
$$

and the internal masses can have a non-positive imaginary part

$$
m_{i}^{2}=m_{i}^{2}-i m_{i} \Gamma_{i}, \quad m_{i} \Gamma_{i} \geq 0, \quad i=1,2,3,4
$$

with $\Gamma_{i}$ are the width of the internal particles. The Feynman parameter representation reads

$$
\begin{aligned}
D_{0} & =\int_{0}^{\infty} d x_{1} d x_{2} d x_{3} d x_{4} \frac{\delta\left(\sum_{i=1}^{4} x_{i}-1\right)}{\Delta^{2}} \\
\Delta & =\frac{1}{2} \sum_{i, j=1}^{4} x_{i} x_{j} Q_{i j}-i \epsilon, \quad Q_{i j}=m_{i}^{2}+m_{j}^{2}-\left(k_{i}-k_{j}\right)^{2} .
\end{aligned}
$$


$Q_{i j}$ is called the Landau matrix. By changing the integration variables as $t=\sum_{i=1}^{4} x_{i}$, $x=\sum_{i=1}^{3} x_{i}, y=x_{1}+x_{2}, z=x_{1}$ and integrating out the Dirac delta function we obtain

$$
\begin{aligned}
D_{0} & =\int_{0}^{1} d x \int_{0}^{x} d y \int_{0}^{y} d z \\
& \times \frac{1}{\left(a x^{2}+b y^{2}+g z^{2}+c x y+h x z+j y z+d x+e y+k z+f-i \epsilon\right)^{2}}
\end{aligned}
$$

with

$$
\begin{aligned}
& a=p_{3}^{2}, \quad b=p_{2}^{2}, \quad g=p_{1}^{2}, \quad c=2 p_{2} \cdot p_{3}, \quad h=2 p_{1} \cdot p_{3}, \quad j=2 p_{1} \cdot p_{2}, \\
& d=m_{3}^{2}-m_{4}^{2}-p_{3}^{2}, \quad e=m_{2}^{2}-m_{3}^{2}-p_{2}^{2}-2 p_{2} \cdot p_{3}, \quad k=m_{1}^{2}-m_{2}^{2}+p_{1}^{2}+2 p_{1} \cdot p_{4}, \\
& f=m_{4}^{2} .
\end{aligned}
$$

It is important to notice that $a, b, g, c, h$ and $j$ are real while $d, e, k$ and $f$ are complex. The denominator always has a negative imaginary part as indicated by the " $-i \epsilon "$

$$
\operatorname{Im}(d x+e y+k z+f-i \epsilon)=\operatorname{Im}\left[z m_{1}^{2}+(y-z) m_{2}^{2}+(x-y) m_{3}^{2}+(1-x) m_{4}^{2}-i \epsilon\right]<0 .
$$

The Landau determinant as a function of new variables reads

$$
\begin{aligned}
\operatorname{det}(Q)= & k^{2} c^{2}+e^{2} h^{2}+d^{2} j^{2}-2 e h k c-2 d j k c-2 d e h j \\
& +4 c d e g+4 c f h j-4 f g c^{2}-4 a f j^{2}-4 a e^{2} g+4 a e j k \\
& -4 b g d^{2}+4 b h k d-4 b f h^{2}-4 b a k^{2}+16 b a f g .
\end{aligned}
$$

The scalar one-loop four-point integral is a function of 10 variables:

$$
D_{0}=D_{0}\left(a_{1}, a_{2}, a_{3}, a_{4}, a_{5}, a_{6} ; b_{1}, b_{2}, b_{3}, b_{4}\right)
$$

where the set of variables are given in Table 1 which shows all the symmetries of this functiont Using those symmetries, we see that the notation of external momentum is extended to include also $\left(p_{1}+p_{2}\right)$ and $\left(p_{2}+p_{3}\right)$. This definition of external momentum will be used hereafter.

\subsection{At least one lightlike momentum}

If there exists at least one lightlike momentum which we can always choose to be $p_{1}$ then the denominator in Eq. (5) becomes linear in $z$. The last integral can be easily performed to get

$$
D_{0}^{(1)}=\mathcal{T}(a, b, c, h, j ; d, e, f, k)-\mathcal{T}(a, b+j, c+h, h, j ; d, e+k, f, k),
$$

where the calculation of the $\mathcal{T}$ function is given in Subsection 2.3. The result contains 72 Spence functions since each $\mathcal{T}$ function contains 36 Spence functions.

In the case where $D_{0}$ has two non-adjacent lightlike momenta, say $p_{1}^{2}=p_{3}^{2}=0$, the result can be written in terms of two $\mathcal{T}$ functions as above. However, one should rather

\footnotetext{
${ }^{\dagger}$ Eq. (4) shows that $D_{0}$ has $4 !=24$ symmetries.
} 
Table 1: Symmetries of $D_{0}$

\begin{tabular}{|c|c|c|c|c|c|c|c|c|c|}
\hline$a_{1}$ & $a_{2}$ & $a_{3}$ & $a_{4}$ & $a_{5}$ & $a_{6}$ & $b_{1}$ & $b_{2}$ & $b_{3}$ & $b_{4}$ \\
\hline$\overline{p_{1}^{2}}$ & 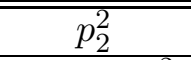 & $\overline{p_{3}^{2}}$ & 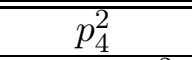 & $\overline{\left(\overline{\left(p_{1}+p_{2}\right)^{2}}\right.}$ & $\overline{\left(p_{2}+p_{3}\right)^{2}}$ & 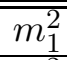 & $\overline{m_{2}^{2}}$ & $\overline{m_{3}^{2}}$ & $m_{4}^{2}$ \\
\hline$p_{1}^{2}$ & $\left(p_{2}+p_{3}\right)^{2}$ & $p_{3}^{2}$ & $\left(p_{1}+p_{2}\right)^{2}$ & $p_{4}^{2}$ & $p_{2}^{2}$ & $m_{1}^{2}$ & $m_{2}^{2}$ & $m_{4}^{2}$ & $m_{3}^{2}$ \\
\hline$\left(p_{1}+p_{2}\right)^{2}$ & $p_{2}^{2}$ & $\left(p_{2}+p_{3}\right)^{2}$ & $p_{4}^{2}$ & $p_{1}^{2}$ & $p_{3}^{2}$ & $m_{1}^{2}$ & $m_{3}^{2}$ & $m_{2}^{2}$ & $m_{4}^{2}$ \\
\hline$\left(p_{1}+p_{2}\right)^{2}$ & $p_{3}^{2}$ & $\left(p_{2}+p_{3}\right)^{2}$ & $p_{1}^{2}$ & $p_{4}^{2}$ & $p_{2}^{2}$ & $m_{1}^{2}$ & $m_{3}^{2}$ & $m_{4}^{2}$ & $m_{2}^{2}$ \\
\hline$p_{4}^{2}$ & $\left(p_{2}+p_{3}\right)^{2}$ & $p_{2}^{2}$ & $\left(p_{1}+p_{2}\right)^{2}$ & $p_{1}^{2}$ & $p_{3}^{2}$ & $m_{1}^{2}$ & $m_{4}^{2}$ & $m_{2}^{2}$ & $m_{3}^{2}$ \\
\hline$p_{4}^{2}$ & $p_{3}^{2}$ & $p_{2}^{2}$ & $p_{1}^{2}$ & $\left(p_{1}+p_{2}\right)^{2}$ & $\left(p_{2}+p_{3}\right)^{2}$ & $m_{1}^{2}$ & $m_{4}^{2}$ & $m_{3}^{2}$ & $m_{2}^{2}$ \\
\hline$p_{1}^{2}$ & $\left(p_{1}+p_{2}\right)^{2}$ & $p_{3}^{2}$ & $\left(p_{2}+p_{3}\right)^{2}$ & $p_{2}^{2}$ & $p_{4}^{2}$ & $m_{2}^{2}$ & $m_{1}^{2}$ & $m_{3}^{2}$ & $m_{4}^{2}$ \\
\hline$p_{1}^{2}$ & $p_{4}^{2}$ & $p_{3}^{2}$ & $p_{2}^{2}$ & $\left(p_{2}+p_{3}\right)^{2}$ & $\left(p_{1}+p_{2}\right)^{2}$ & $m_{2}^{2}$ & $m_{1}^{2}$ & $m_{4}^{2}$ & $m_{3}^{2}$ \\
\hline$p_{2}^{2}$ & $\left(p_{1}+p_{2}\right)^{2}$ & $p_{4}^{2}$ & $\left(p_{2}+p_{3}\right)^{2}$ & $p_{1}^{2}$ & $p_{3}^{2}$ & $m_{2}^{2}$ & $m_{3}^{2}$ & $m_{1}^{2}$ & $m_{4}^{2}$ \\
\hline$p_{2}^{2}$ & $p_{3}^{2}$ & $p_{4}^{2}$ & $p_{1}^{2}$ & $\left(p_{2}+p_{3}\right)^{2}$ & $\left(p_{1}+p_{2}\right)^{2}$ & $m_{2}^{2}$ & $m_{3}^{2}$ & $m_{4}^{2}$ & $m_{1}^{2}$ \\
\hline$\left(p_{2}+p_{3}\right)^{2}$ & $p_{4}^{2}$ & $\left(p_{1}+p_{2}\right)^{2}$ & $p_{2}^{2}$ & $p_{1}^{2}$ & $p_{3}^{2}$ & $m_{2}^{2}$ & $m_{4}^{2}$ & $m_{1}^{2}$ & $m_{3}^{2}$ \\
\hline$\left(p_{2}+p_{3}\right)^{2}$ & $p_{3}^{2}$ & $\left(p_{1}+p_{2}\right)^{2}$ & $p_{1}^{2}$ & $p_{2}^{2}$ & $p_{4}^{2}$ & $m_{2}^{2}$ & $m_{4}^{2}$ & $m_{3}^{2}$ & $m_{1}^{2}$ \\
\hline$\left(p_{1}+p_{2}\right)^{2}$ & $p_{1}^{2}$ & $\left(p_{2}+p_{3}\right)^{2}$ & $p_{3}^{2}$ & $p_{2}^{2}$ & $p_{4}^{2}$ & $m_{3}^{2}$ & $m_{1}^{2}$ & $m_{2}^{2}$ & $m_{2}^{2}$ \\
\hline$\left(p_{1}+p_{2}\right)^{2}$ & $p_{4}^{2}$ & $\left(p_{2}+p_{3}\right)^{2}$ & $p_{2}^{2}$ & $p_{3}^{2}$ & $p_{1}^{2}$ & $m_{3}^{2}$ & $m_{1}^{2}$ & $m_{4}^{2}$ & $m_{2}^{2}$ \\
\hline$p_{2}^{2}$ & $p_{1}^{2}$ & $p_{4}^{2}$ & $p_{3}^{2}$ & $\left(p_{1}+p_{2}\right)^{2}$ & $\left(p_{2}+p_{3}\right)^{2}$ & $m_{3}^{2}$ & $m_{2}^{2}$ & $m_{1}^{2}$ & $m_{4}^{2}$ \\
\hline$p_{2}^{2}$ & $\left(p_{2}+p_{3}\right)^{2}$ & $p_{4}^{2}$ & $\left(p_{1}+p_{2}\right)^{2}$ & $p_{3}^{2}$ & $p_{1}^{2}$ & $m_{3}^{2}$ & $m_{2}^{2}$ & $m_{4}^{2}$ & $m_{1}^{2}$ \\
\hline$p_{3}^{2}$ & $p_{4}^{2}$ & $p_{1}^{2}$ & $p_{2}^{2}$ & $\left(p_{1}+p_{2}\right)^{2}$ & $\left(p_{2}+p_{3}\right)^{2}$ & $m_{3}^{2}$ & $m_{4}^{2}$ & $m_{1}^{2}$ & $m_{2}^{2}$ \\
\hline$p_{3}^{2}$ & $\left(p_{2}+p_{3}\right)^{2}$ & $p_{1}^{2}$ & $\left(p_{1}+p_{2}\right)^{2}$ & $p_{2}^{2}$ & $p_{4}^{2}$ & $m_{3}^{2}$ & $m_{4}^{2}$ & $m_{2}^{2}$ & $m_{1}^{2}$ \\
\hline$p_{4}^{2}$ & $p_{1}^{2}$ & $p_{2}^{2}$ & $p_{3}^{2}$ & $\left(p_{2}+p_{3}\right)^{2}$ & $\left(p_{1}+p_{2}\right)^{2}$ & $m_{4}^{2}$ & $m_{1}^{2}$ & $m_{2}^{2}$ & $m$ \\
\hline$p_{4}^{2}$ & $\left(p_{1}+p_{2}\right)^{2}$ & $p_{2}^{2}$ & $\left(p_{2}+p_{3}\right)^{2}$ & $p_{3}^{2}$ & $p_{1}^{2}$ & $m_{4}^{2}$ & $m_{1}^{2}$ & $m_{3}^{2}$ & $\overrightarrow{m_{2}^{2}}$ \\
\hline$\left(p_{2}+p_{3}\right)^{2}$ & $p_{1}^{2}$ & $\left(p_{1}+p_{2}\right)^{2}$ & $p_{3}^{2}$ & $p_{4}^{2}$ & $p_{2}^{2}$ & $m_{4}^{2}$ & $m_{2}^{2}$ & $m_{1}^{2}$ & $m_{3}^{2}$ \\
\hline$\left(p_{2}+p_{3}\right)^{2}$ & $p_{2}^{2}$ & $\left(p_{1}+p_{2}\right)^{2}$ & $p_{4}^{2}$ & $p_{3}^{2}$ & $p_{1}^{2}$ & $m_{4}^{2}$ & $m_{2}^{2}$ & $m_{3}^{2}$ & $m_{1}^{2}$ \\
\hline$p_{3}^{2}$ & $\left(p_{1}+p_{2}\right)^{2}$ & $p_{1}^{2}$ & $\left(p_{2}+p_{3}\right)^{2}$ & $p_{4}^{2}$ & $p_{2}^{2}$ & $m_{4}^{2}$ & $m_{3}^{2}$ & $m_{1}^{2}$ & $m ?$ \\
\hline$p_{3}^{2}$ & $p_{2}^{2}$ & $p_{1}^{2}$ & $p_{4}^{2}$ & $\left(p_{2}+p_{3}\right)^{2}$ & $\left(p_{1}+p_{2}\right)^{2}$ & $m_{4}^{2}$ & $m_{3}^{2}$ & $m_{2}^{2}$ & $m_{1}^{2}$ \\
\hline
\end{tabular}

combine them into one single function as (in this way, the result contains a lesser number of Spence functions)

$$
\begin{aligned}
D_{0}^{(13)} & =\int_{0}^{1} d x \int_{0}^{x} d y \\
& \times \frac{y}{\left(G y^{2}+H x y+D x+I y+F-i \epsilon\right)\left(A y^{2}+C x y+D x+E y+F-i \epsilon\right)}
\end{aligned}
$$

where

$$
\begin{array}{r}
A=b+j, \quad C=c+h, \quad D=d, \quad E=e+k, \\
F=f, \quad G=b, \quad H=c, \quad I=e .
\end{array}
$$

We notice that each denominator is a linear function in $x$ hence one can perform the $x$-integral by using:

$$
\int_{0}^{1} d x \int_{0}^{x} d y=\int_{0}^{1} d y \int_{y}^{1} d x
$$


The result is written in terms of 32 Spence functions [3, 6]

$$
\begin{aligned}
D_{0}^{(13)} & =\int_{0}^{1} \frac{d y}{S V-T U}\left(\ln \frac{S+T}{U+V}-\ln \frac{S y+T}{U y+V}\right) \\
& =\frac{1}{(H A-C G)\left(y_{2}-y_{1}\right)} \sum_{i=1}^{2} \sum_{j=1}^{4}(-1)^{i+j} \int_{0}^{1} \frac{d y}{y-y_{i}} \ln \left(A_{j} y^{2}+B_{j} y+C_{j}\right),
\end{aligned}
$$

where

$$
\begin{aligned}
& S=H y+D, \quad T=G y^{2}+I y+F-i \epsilon \\
& U=C y+D, \quad V=A y^{2}+E y+F-i \epsilon
\end{aligned}
$$

and $y_{1,2}$ are the two roots of the equation

$$
(H A-C G) y^{2}+(A D+H E-D G-C I) y+D E-D I+(H-C)(F-i \epsilon)=0
$$

Or用

$$
y_{1,2}=\frac{-(A D+H E-D G-C I) \mp \sqrt{\operatorname{det}(Q)}}{2(H A-C G)}
$$

where the indices 1, 2 correspond to - and + signs respectively and the discriminant,

$$
\operatorname{det}(Q)=(A D+H E-D G-C I)^{2}-4(H A-C G)(D E-D I+H F-C F),
$$

is nothing but the determinant of the Landau matrix $Q$ defined in Eq. (8). The various coefficients of the argument of the logarithms are given in Table2. The integral in Eq. (14) will be considered in Appendix B.

Table 2: The coefficients of the $D_{0}^{(13)}$-function

\begin{tabular}{cccc}
\hline$j$ & $A_{j}$ & $B_{j}$ & $C_{j}$ \\
\hline 1 & $A$ & $E+C$ & $D+F-i \epsilon$ \\
2 & $G$ & $I+H$ & $D+F-i \epsilon$ \\
3 & $G+H$ & $D+I$ & $F-i \epsilon$ \\
4 & $A+C$ & $E+D$ & $F-i \epsilon$ \\
\hline
\end{tabular}

\subsection{General case}

As proven in [7] (see Eq. (6.18)), $D_{0}$ can be written as

$$
\begin{aligned}
D_{0} & =-\mathcal{T}(a+b+c, g, j+h, c+2 b+h \alpha+j \alpha, j+2 \alpha g ; d+e, k, f, e+k \alpha) \\
& +(1-\alpha) \mathcal{T}(a, b+g+j, c+h, c+h \alpha,(j+2 \alpha g)(1-\alpha) ; d, e+k, f, e+k \alpha) \\
& +\alpha \mathcal{T}(a, b, c, c+h \alpha,-(j+2 \alpha g) \alpha ; d, e, f, e+k \alpha)
\end{aligned}
$$

\footnotetext{
${ }^{\ddagger}$ In the case of real masses, the " $i \epsilon "$-part of each root should be written separately as shown in Eq. (A.2).
} 
where $\alpha$ is a root of the equation

$$
g \alpha^{2}+j \alpha+b=0 .
$$

The method is restricted to real $\alpha$, i.e.

$$
\begin{aligned}
j^{2}-4 b g & =4\left[\left(p_{1} \cdot p_{2}\right)^{2}-p_{1}^{2} p_{2}^{2}\right] \\
& =\lambda\left[\left(p_{1}+p_{2}\right)^{2}, p_{1}^{2}, p_{2}^{2}\right] \geq 0, \\
\lambda(x, y, z) & =x^{2}+y^{2}+z^{2}-2 x y-2 x z-2 y z .
\end{aligned}
$$

This is satisfied if $p_{1}$ (or $p_{2}$ or $p_{1}+p_{2}$ ) is timelike. If the above condition is not fulfilled then one has to keep rotating the external momenta by using the symmetric properties given in Table 1 until it is met. The right hand side (rhs) of Eq. (19) contains $36 \times 3=108$ Spence functions.

\subsection{The $\mathcal{T}$ function}

We now consider the following $\mathcal{T}$ function

$$
\begin{aligned}
\mathcal{T}(A, B, C, G, H & ; D, E, F, J)=\int_{0}^{1} d x \int_{0}^{x} d y \\
& \times \frac{1}{(G x+H y+J)\left(A x^{2}+B y^{2}+C x y+D x+E y+F-i \epsilon\right)},
\end{aligned}
$$

where $A, B, C, G$ and $H$ are real and $D, E, F$ and $J$ can be complex with the restriction that the imaginary part of the second denominator has a definite sign, say negative as indicated by the $-i \epsilon$. In order to make the imaginary part of the first denominator also negative, we multiply both the numerator and denominator of the integrand with $-s_{J}=-\operatorname{sign}(J)$ and get

$$
\mathcal{T}=-s_{J} \int_{0}^{1} d x \int_{0}^{x} d y \frac{1}{\left(G x+H y+J-i \epsilon^{\prime}\right)\left(A x^{2}+B y^{2}+C x y+D x+E y+F-i \epsilon\right)}
$$

where $G=-s_{J} G, H=-s_{J} H, J=-s_{J} J$ and $\epsilon^{\prime}$ is infinitesimally positive.

2.3.1 General case: $A \neq 0$ and $B \neq 0$

Following the method explained in [7], we obtain (similar to Eq. (6.26) of [7])

$$
\begin{aligned}
-s_{J} \mathcal{T} & =\frac{1}{K\left(y_{2}-y_{1}\right)} \sum_{i=1}^{2} \sum_{j=1}^{6}(-1)^{i} \int_{0}^{1} d y \frac{c_{j}}{(-1)^{j+1} c_{j} y-b_{j}-y_{i}} \ln \left(A_{j} y^{2}+B_{j} y+C_{j}\right) \\
& -\frac{1}{K\left(y_{2}-y_{1}\right)} \sum_{i=1}^{2}(-1)^{i} P_{i} \ln R_{i}
\end{aligned}
$$

with the following cascade of notation.

$$
y_{1}=\frac{-L-\sqrt{\operatorname{det}(Q)}}{2 K}, \quad y_{2}=\frac{-L+\sqrt{\operatorname{det}(Q)}}{2 K}, \quad \operatorname{det}(Q)=L^{2}-4 N K
$$


are the roots and the discriminant of the equation

$$
\begin{aligned}
K y^{2}+L y+N & =0, \quad K=B(G+\beta H)-H(C+2 \beta B), \\
L & =(G+\beta H) E-H(D+\beta E)-J(C+2 \beta B), \\
N & =(G+\beta H)(F-i \epsilon)-(D+\beta E) J
\end{aligned}
$$

with $\beta$ is one root of the equation

$$
B \beta^{2}+C \beta+A=0 .
$$

It is easy to check that the discriminant is free of $\beta$ and is indeed the determinant of the Landau matrix $Q$ if our calculation is set in the context of Eq. (10) or Eq. (19). And

$$
\begin{aligned}
R_{i} & =\frac{H y_{i}+J}{B y_{i}^{2}+E y_{i}+F-i \epsilon}=\frac{G+\beta H}{(C+2 \beta B) y_{i}+D+\beta E}, \\
P_{i} & =\sum_{j=1}^{3} \int_{0}^{1} d y \frac{c_{j}}{(-1)^{j+1} c_{j} y-b_{j}-y_{i}}=\oint_{\triangle_{\beta}} d y \frac{1}{y-y_{i}},
\end{aligned}
$$

where $\triangle_{\beta}$ is the beta triangle $[0,1-\beta,-\beta]$ as shown in Fig. 2, The second term in Eq. (25) is nothing but the residue contribution. All the other coefficients are listed in

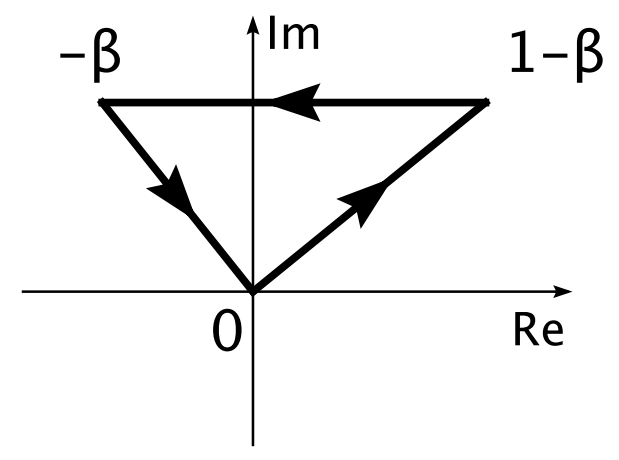

Figure 2: The beta triangle $[0,1-\beta,-\beta]$ in the complex plane.

Table 3.

Table 3: The coefficients of the $\mathcal{T}$ function

\begin{tabular}{cccccc}
\hline$j$ & $c_{j}$ & $b_{j}$ & $A_{j}$ & $B_{j}$ & $C_{j}$ \\
\hline 1 & 1 & $\beta$ & 0 & $H$ & $G+J-i \epsilon^{\prime}$ \\
2 & $-1+\beta$ & 0 & 0 & $G+H$ & $J-i \epsilon^{\prime}$ \\
3 & $-\beta$ & 0 & 0 & $G$ & $J-i \epsilon^{\prime}$ \\
4 & -1 & $\beta$ & $B$ & $C+E$ & $A+D+F-i \epsilon$ \\
5 & $1-\beta$ & 0 & $A+B+C$ & $E+D$ & $F-i \epsilon$ \\
6 & $\beta$ & 0 & $A$ & $D$ & $F-i \epsilon$ \\
\hline
\end{tabular}


We have an important remark here. Eq. (25) is very different from Eq. (6.26) of [7] in the respect of numerical evaluation. 't Hooft and Veltman suggested a way to proceed with the latter by splitting the residue into two parts: one part has the same form as the first term in Eq. (25) but with the variable $y$ in the argument of the logarithms replaced by the pole of the corresponding previous factor, the other part is related to the $\eta$-function. Eq. (6.26) of [7] has two disadvantages. First, this equation is used in connection with the function $\mathcal{S}_{3}$ defined in Appendix B of [7]. However, $\mathcal{S}_{3}$ is only well defined if $y_{0}$ is not a root of the argument of the second logarithm. In fact, there are several cases where the second logarithm of $\mathcal{S}_{3}$ is divergent but the final result of $\mathcal{T}$ is finite. This point is made clear from the calculation of the function $\mathcal{S}_{1}$ in Appendix B. Second, this equation leads to the evaluation of several $\eta$-functions which might suffer from nasty cancellations. In particular, the $\eta$-functions in eqs. (6.26, B.2) of [7] may cause serious problems.

The representation (8) leads naturally to two ways of numerical evaluation. This will be discussed in Section 3 .

\subsubsection{Special cases: $A=0$ or $B=0$}

These special cases can occur when we apply Eq. (10) for the case of more than one lightlike momenta.

If $B=0$ then both denominators in Eq. (24) are linear in $y$ then the $y$-integral can be easily performed. The result reads

$$
\begin{aligned}
\mathcal{T}(A, 0, C, G, H ; D, E, F, J) & =\frac{1}{(H A-C G)\left(y_{2}-y_{1}\right)} \sum_{i=1}^{2} \sum_{j=1}^{4}(-1)^{i+j} \\
& \times \int_{0}^{1} d y \frac{1}{y-y_{i}} \ln \left(A_{j} y^{2}+B_{j} y+C_{j}\right),
\end{aligned}
$$

where $y_{1,2}$ are the two roots of

$$
(H A-C G) y^{2}+(H D-C J-E G) y+H(F-i \epsilon)-E\left(J-i \epsilon^{\prime}\right)=0
$$

or

$$
\begin{aligned}
y_{1} & =\frac{-(H D-C J-E G)-\sqrt{\operatorname{det}(Q)}}{2(H A-C G)}, y_{2}=\frac{-(H D-C J-E G)+\sqrt{\operatorname{det}(Q)}}{2(H A-C G)} \\
\operatorname{det}(Q) & =(H D-C J-E G)^{2}-4(H A-C G)(H F-E J) .
\end{aligned}
$$

The discriminant is the determinant of the Landau matrix $Q$ if our calculation is set in the context of Eq. (10) or Eq. (19). The other coefficients are given in Table 4 ,

If $A=0$, we change the integration order by using Eq. (13) and then change the integration variables in two successive steps: $x=x^{\prime}+y$ and $y=1-y^{\prime}$. At the end, one should obtain the following relation

$$
\begin{aligned}
\mathcal{T}(0, B, C, G, H & ; D, E, F, J)=\mathcal{T}(B+C, 0,-C,-G-H, G ; \\
& -D-E-2 B-2 C, D+C, D+E+B+C+F, G+H+J) .
\end{aligned}
$$

Thus, we get back to the above case of $B=0$. 
Table 4: The coefficients of the $\mathcal{T}$ function

\begin{tabular}{cccc}
\hline$j$ & $A_{j}$ & $B_{j}$ & $C_{j}$ \\
\hline 1 & $A+C$ & $E+D$ & $F-i \epsilon$ \\
2 & 0 & $G+H$ & $J-i \epsilon^{\prime}$ \\
3 & 0 & $G$ & $J-i \epsilon^{\prime}$ \\
4 & $A$ & $D$ & $F-i \epsilon$ \\
\hline
\end{tabular}

We conclude this section by observing that the above results can be also applied for the case of real masses. In this case, the two poles $y_{1,2}$ in the rhs of eqs. (14, 25, 30) can be real and the $i \epsilon$-prescription is needed. The position of the poles depend on the value of internal masses and external momenta. Thus, it might happen that the two poles become equal and pinch the integration contour, $[0,1]$. If this occurs then the $D_{0}$ function can have a four-point leading Landau singularity (LLS). The issue of Landau singularities will be discussed in the appendices.

\section{The code DOC}

The above method has been implemented into a Fortran 77 code, DoC, which can calculate the scalar one-loop four-point integral, $D_{0}$, with complex/real internal masses. The restriction is that at least one external momentum must be lightlike or $\alpha$ must be real (see Eq. (21) ). The code is best exploited when included into the library LoopTools where a reduction procedure to calculate tensor one-loop five-point integrals with complex masses is available. Thus, the new version of LoopTools (LoopTools-2.4) can handle all the one-loop integrals with complex masses up to five-point. The original code DOC can be downloaded at:

$$
\text { http://wwwth.mppmu.mpg.de/members/ldninh/index.html }
$$

LoopTools-2.4 including Dod§ can be downloaded at:

$$
\text { http://www.feynarts.de/looptools/ }
$$

In the language of LoopTools, the $D_{0}$ function with complex masses, also named DOC, is invoked in the same way as the usual DO with real masses. However, all the arguments of DOC must be of double complex type.

The implementation of Eq. (25) is straightforward. However, there is an important point we want to stress. The residue term on the rhs may cause serious problem of numerical cancellation when the imaginary part of the $R_{i}$ s become very small. Thus we want to eliminate this term as far as possible. Our algorithm is based on the simple fact that this contribution vanishes if the pole is outside of the beta triangle. If $\beta$ is real, the triangle becomes a line in the complex plane. The residue contribution vanishes. If $\beta$ is complex, there are two choices. First, we take $\beta=\beta_{1}$ which is the largest root of Eq. (28).

\footnotetext{
$\S$ Thomas Hahn has adapted this code to LoopTools. The structure is unchanged. The way of coding is slightly modified. The numerical results of the two codes should be identical.
} 
There is a very efficient method to check whether a point (pole) is inside a triangle by using the barycentric technique. This idea is very simple. From a triangle, ABC, one can define a barycentric reference frame $(\overrightarrow{A B}, \overrightarrow{A C})$ where $\mathrm{A}$ is the origin. The coordinates of a point $\mathrm{P},(x, y)$, are easily calculated by writing:

$$
\overrightarrow{A P}=x \overrightarrow{A B}+y \overrightarrow{A C}
$$

Multiply both sides by $\overrightarrow{A B}$ and $\overrightarrow{A C}$ to get a system of two equations to solve for $(x, y)$. The conditions for $\mathrm{P}$ to be inside the triangle $\mathrm{ABC}$ are: $x$ and $y$ must be positive and $x+y \leq 1$. However, one sees that the problem of numerical cancellation is still present if $x$ or $y$ is very small, i.e. the point $\mathrm{P}$ is very close to the border of the triangle. If this happens, we repeat the above steps with the second choice $\beta=\beta_{2}$. That is what we have done in the code.

In the worst case, if $x$ or $y$ is very small for both choices of $\beta$ then the code will produce a warning of numerical cancellation but still continue the computation. The result in this case is not reliable and must be cross-checked. The code includes a means to do this. Indeed, we have written two different codes, called versions 1 and 2 , to evaluate the rhs of Eq. (25) based on two different strategies. The first one is described as above. In the second one, the residue contribution is always calculated as long as $\beta$ is complex. One has to specify which version when calling the main function:

$$
\mathrm{d} 0 \mathrm{c}=\mathrm{D} 0 \mathrm{Cn}(\mathrm{meth}, \mathrm{p} 1, \mathrm{p} 2, \mathrm{p} 3, \mathrm{p} 4, \mathrm{p} 1 \mathrm{p} 2, \mathrm{p} 2 \mathrm{p} 3, \mathrm{~m} 1, \mathrm{~m} 2, \mathrm{~m} 3, \mathrm{~m} 4)
$$

where meth $=0$ (version 1 ) or 1 (version 2 ). The two versions are different only when $\beta$ is complex. For LoopTools-2.4, one can choose different versions by defining a version key before calling the DOC function as

$$
\begin{aligned}
& \text { call setversionkey }\left(\mathrm{i}^{*} \operatorname{KeyD} 0 \mathrm{C}\right) \\
& \mathrm{d} 0 \mathrm{c}=\mathrm{D} 0 \mathrm{C}(\mathrm{p} 1, \mathrm{p} 2, \mathrm{p} 3, \mathrm{p} 4, \mathrm{p} 1 \mathrm{p} 2, \mathrm{p} 2 \mathrm{p} 3, \mathrm{~m} 1, \mathrm{~m} 2, \mathrm{~m} 3, \mathrm{~m} 4)
\end{aligned}
$$

where $i$ can be 0 (compute version 1), 1 (compute version 2), 2 (compute both versions, return 1) or 3 (compute both versions, return 2). The default value is $i=0$.

The structure of the original code, written in double precision Fortran 77, is as follows.

- DoCn.F: the main function to return the value of the integral $D_{0}$ as a function of masses and momenta (see Eq. (11)). It rotates the momenta to the right position and prepares input parameters for Eq. (5).

- d0c_abc.F: a subroutine called in DOCn.F to calculate the rhs of Eq. (5).

- $\mathrm{fT13.F}$ is a subroutine to calculate the function $D_{0}^{(13)}$ as defined by Eq. (11).

- $f T n . F\left(i n c l u d i n g ~ f T n \_l i n . F\right.$ to handle special cases) is a subroutine to calculate the $\mathcal{T}$ function (defined by Eq. (23) ) by using the barycentric technique described above. It belongs to version 1.

- $f T n \_r e s d . F\left(\right.$ including $f T n \_l i n . F$ to handle special cases) is another subroutine to calculate the $\mathcal{T}$ function for cross-checking purpose. It belongs to version 2 .

- fS3n.F and fR.F are subroutines to calculate the functions $\mathcal{S}_{1}$ and $\mathcal{R}_{1}$ defined in Appendix B. 
- $\mathrm{fS} 2 . \mathrm{F}$ is a subroutine to calculate the functions $\mathcal{T}$ and $D_{0}^{(13)}$ if the integrand is just a logarithm, i.e. the prefactor is just a constant.

- Fundamental functions including Spence function, logarithm, $\eta$-function are taken from $\mathrm{FF}$.

The code has been carefully checked as follows. Since the $i \epsilon$-prescription is kept explicitly in our code, the code works also for the case of real masses. In this case, our results have been checked by comparing with the D0 function of LoopTools. Full agreement with the version 'b' of LoopTools has been found 1 . We then introduced the widths for internal particles and checked the results in two limits: very small and very large widths. In the former case, we recovered the results of real masses. For the latter case, the results were checked against the ones of a routine where the integration is done numerically by using BASES [13]. We observed good agreement within the integration error (see Table 5).

Tables 6,7,8 give some numerical results of the code for two cases: complex and real masses. For the latter we observed that our code agrees with version 'b' of LoopTools while disagrees with version 'a'. In fact, our code produces the same results with both versions of LoopTools in almost all physical cases. The results shown in Tables 7, 8 are a few cases where disagreement has been found I. Thus, care must be taken when using loop libraries and one should always cross-check the results by using different methods. For instance, it can be very useful to check the results of real masses in a tricky situation by using the code for complex masses with a very small imaginary part.

Table 5: Comparisons with a numerical integration routine using BASES, complex masses with large imaginary parts: $m_{1}^{2}=28900-25500 i, m_{2}^{2}=6400-16000 i, m_{3}^{2}=8100-22500 i$, $m_{4}^{2}=40000-80000 i, p_{1}^{2}=100, p_{2}^{2}=32400, p_{3}^{2}=400 p_{4}^{2}=250000,\left(p_{2}+p_{3}\right)^{2}=84100$. Numerical integration errors are about $(0.03 \%, 0.07 \%)$.

\begin{tabular}{ccc}
\hline$\left(p_{1}+p_{2}\right)^{2}$ & DOC & Numerical integration \\
\hline 48400 & $(-1.50823957 \mathrm{E}-10,1.62902558 \mathrm{E}-11)$ & $(-1.5077496 \mathrm{E}-10,1.63063608 \mathrm{E}-11)$ \\
52900 & $(-1.51384299 \mathrm{E}-10,1.37108163 \mathrm{E}-11)$ & $(-1.51339092 \mathrm{E}-10,1.37149562 \mathrm{E}-11)$ \\
57600 & $(-1.51834347 \mathrm{E}-10,1.09709949 \mathrm{E}-11)$ & $(-1.51787895 \mathrm{E}-10,1.09683749 \mathrm{E}-11)$ \\
62500 & $(-1.52150627 \mathrm{E}-10,8.07814957 \mathrm{E}-12)$ & $(-1.52101762 \mathrm{E}-10,8.07660917 \mathrm{E}-12)$ \\
\hline
\end{tabular}

\footnotetext{
'LoopTools has two versions, 'a' and 'b', to calculate the D0 function [11. Version 'a' is based on FF while version 'b' uses the method of 12 .

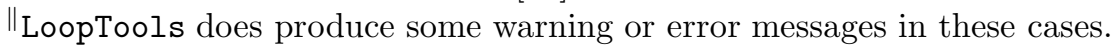


Table 6: Some results of DOC, complex masses with small imaginary parts: $m_{1}^{2}=28900-$ $255 i, m_{2}^{2}=6400-160 i, m_{3}^{2}=8100-225 i, m_{4}^{2}=40000-800 i, p_{4}^{2}=250000,\left(p_{1}+p_{2}\right)^{2}=$ 72900, $\left(p_{2}+p_{3}\right)^{2}=84100$

\begin{tabular}{cccc}
\hline$p_{1}^{2}$ & $p_{2}^{2}$ & $p_{3}^{2}$ & $\mathrm{DOC} \times 10^{-10}$ \\
\hline 100 & 32400 & 400 & $(-37.6230835,-134.501524)$ \\
0 & 32400 & 400 & $(-37.8749852,-134.892905)$ \\
0 & 32400 & 0 & $(-38.6796394,-136.041222)$ \\
0 & 0 & 0 & $(-28.9137581,16.3570695)$ \\
\hline
\end{tabular}

Table 7: Comparisons between LoopTools and DoC, real masses: $m_{1}^{2}=m_{2}^{2}=m_{3}^{2}=m_{4}^{2}=$ $30276, p_{4}^{2}=14400,\left(p_{1}+p_{2}\right)^{2}=57600$

\begin{tabular}{ccc}
\hline$\left(p_{1}^{2}, p_{2}^{2}, p_{3}^{2},\left(p_{2}+p_{3}\right)^{2}\right)$ & $(0,124609,20,129630)$ & $(0,0,20,73473)$ \\
\hline LoopTools 'a' $\times 10^{-10}$ & $(-8.4106454,227.290811)$ & $(19.8584112,-8.23191831)$ \\
\hline LoopTools 'b' $\times 10^{-10}$ & $(-8.4106454,26.7103536)$ & $(3.32391023,-6.755152 \mathrm{E}-16)$ \\
\hline D0C $\times 10^{-10}$ & $(-8.4106454,26.7103536)$ & $(3.32391023,-2.067952 \mathrm{E}-15)^{* *}$ \\
\hline
\end{tabular}

Table 8: Continuation of Table 7

\begin{tabular}{ccc}
\hline$\left(p_{1}^{2}, p_{2}^{2}, p_{3}^{2},\left(p_{2}+p_{3}\right)^{2}\right)$ & $(0,0,0,734730)$ & $(100,50,20,734730)$ \\
\hline LoopTools 'a' $\times 10^{-10}$ & $(-0.400586866,-6.57420392)$ & $(-0.401370928,-6.57507623)$ \\
\hline LoopTools 'b' $\times 10^{-10}$ & $(-0.400586866,1.85027075)$ & $(-0.401370928,1.85083346)$ \\
\hline D0C $\times 10^{-10}$ & $(-0.400586866,1.85027075)$ & $(-0.401370928,1.85083346)$ \\
\hline
\end{tabular}

\section{Conclusions}

The Fortran code DOC allows to calculate the scalar one-loop four-point function with complex masses for all physical processes at colliders. The method and structure of the code were explained. The results related to massless external particles and the discussion of Landau singularities complement the study of 't Hooft and Veltman. The code has been included in LoopTools-2.4. Please refer to this paper if you use the code. Bug reports or comments should be sent to the authors

\section{Acknowledgments}

We thank F. Boudjema for helpful discussions and for careful reading of the manuscript. A. Denner is acknowledged for useful discussions and for checking some results with real masses. Special thanks go to T. Hahn for valuable suggestions, comments and his careful

** The imaginary part of $D_{0}$ is zero in this case since no normal-threshold-cut is open.

${ }^{\dagger}$ Email: ldninh@mppmu.mpg.de or tdao@mppmu.mpg.de 
reading of the manuscript. Indeed, he has spent a lot of time and effort to improve and adapt our code to LoopTools-2.4, thereby making the code more useful for practical calculations. Figures were produced with JaxoDraw [14].

\section{Appendices}

\section{A Four-point leading Landau singularity}

The method of this paper can also be applied for the case of real internal masses. In this case the $i \epsilon$-prescription is needed to define various multivalued functions (logarithms, Spence functions) in the final results. From the practical viewpoint, this method is not as efficient as other methods based on projective transformation [7, 12] since the results are written in terms of a larger number of Spence functions. However, it has some useful virtues as a direct calculation and we will show that this method is indeed a very easy means to understand the analytical properties of the scalar four-point function. To illustrate this purpose, we first consider the following function

$$
\mathcal{T}_{1}=\int_{0}^{1} d y \frac{\ln \left(A y^{2}+B y+C-i \epsilon\right)}{K y^{2}+L y+N-i \epsilon^{\prime}} .
$$

An important property of this function is that the denominator has, in general, two poles

$$
\begin{aligned}
& y_{1}=\frac{-L-\sqrt{L^{2}-4 K N}}{2 K}-\frac{i \epsilon^{\prime}}{\operatorname{Re} \sqrt{L^{2}-4 K N}}, \\
& y_{2}=\frac{-L+\sqrt{L^{2}-4 K N}}{2 K}+\frac{i \epsilon^{\prime}}{\operatorname{Re} \sqrt{L^{2}-4 K N}} .
\end{aligned}
$$

If these poles are real then it may occur that, in some region of parameter space, they pinch the integration contour $[0,1]$, leading to a singularity. This phenomenon occurs when the two roots become equa $\$$, i.e. $\operatorname{det}(Q)=L^{2}-4 K N \rightarrow 0$. Since $y_{2}-y_{1} \propto 2 i \epsilon^{\prime} \neq 0$, we can split $\mathcal{T}_{1}$ into two terms as usual

$$
\mathcal{T}_{1}=\frac{1}{K\left(y_{2}-y_{1}\right)} \int_{0}^{1} d y\left(\frac{1}{y-y_{2}}-\frac{1}{y-y_{1}}\right) \ln \left(A y^{2}+B y+C-i \epsilon\right) .
$$

Using the results of Appendix $\mathrm{B}$ and taking the limit $y_{2} \rightarrow y_{1}=y_{0}=-L /(2 K), y_{0}$ is real, we get

$$
\mathcal{T}_{1}^{\text {sing. }}=\frac{\eta\left(i \epsilon_{1}, i \epsilon_{1}, \frac{i \epsilon_{1}}{y_{0}\left(y_{0}-1\right)}\right)}{\sqrt{\operatorname{det}(Q)}}\left[\ln (A-i \epsilon)+\eta\left(-z_{1},-z_{2}\right)+\mathcal{N}\left(z_{1}, y_{0}\right)+\mathcal{N}\left(z_{2}, y_{0}\right)\right]
$$

where $\epsilon_{1}=\epsilon^{\prime} / \operatorname{Re} \sqrt{L^{2}-4 K N}, z_{1,2}$ are the roots of the argument of the logarithm and

$$
\begin{aligned}
& \mathcal{N}\left(z, y_{0}\right)=\left\{\begin{aligned}
\ln \left(y_{0}-z\right) & \text { if } z \neq y_{0} \\
-\ln \left(-i \epsilon_{z}\right)-\pi i \operatorname{sign}\left(\epsilon_{z}\right) & \text { if } z=y_{0}
\end{aligned}\right. \\
& \eta(a, b, c)=2 \pi i[\theta(-\operatorname{Im} a) \theta(-\operatorname{Im} b) \theta(\operatorname{Im} c)-\theta(\operatorname{Im} a) \theta(\operatorname{Im} b) \theta(-\operatorname{Im} c)] .
\end{aligned}
$$

\footnotetext{
$\ddagger$ This cannot happen in the case of complex masses since the discriminant, the Landau determinant $\operatorname{det}(Q)$, is protected from zero by an imaginary part.
} 
We remark that if

$$
y_{0}\left(y_{0}-1\right)<0, \text { or } 0<y_{0}<1
$$

then the result is divergent. This is the origin of the four-point LLS. We also observe that if $y_{0}=z_{1}$ or/and $z_{2}$ then we have the coincident of four-point and three-point Landau singularities. The latter will be discussed in Appendix B.

The scalar one-loop four-point function, $D_{0}$, is a sum of $\mathcal{T}_{1}$ s. The above conditions for leading Landau singularity are therefore necessary but not sufficient. In fact, the conditions for scalar one-loop integrals to have a LLS can be obtained by using Landau equations [1, 15, 6]. However, one might expect to find out the same conditions from a direct calculation. To illustrate the key points while keeping our explanation as simple as possible, we consider the case of $D_{0}$ function with two opposite lightlike external momenta, see Eq. (14). We ignore the case of coincident singularity and assume $y_{0} \neq z_{1,2}$, Eq. (A.4) becomes

$$
\mathcal{T}_{1}^{\text {sing. }}=\frac{\eta\left(i \epsilon_{1}, i \epsilon_{1}, \frac{i \epsilon_{1}}{y_{0}\left(y_{0}-1\right)}\right)}{\sqrt{\operatorname{det}(Q)}} \ln \left(A y_{0}^{2}+B y_{0}+C-i \epsilon\right),
$$

where we recall that $y_{0}$ is real. Apply this result to Eq. (14) we get

$$
D_{0}^{(13), \text { sing. }}=\frac{\eta\left(i \epsilon_{1}, i \epsilon_{1}, \frac{i \epsilon_{1}}{y_{0}\left(y_{0}-1\right)}\right)}{\sqrt{\operatorname{det}(Q)}}\left(\ln \frac{S_{0}+T_{0}-i \epsilon}{U_{0}+V_{0}-i \epsilon}-\ln \frac{S_{0} y_{0}+T_{0}-i \epsilon}{U_{0} y_{0}+V_{0}-i \epsilon}\right)
$$

where the index 0 in the rhs means that the functions are real (without $i \epsilon$ ) and calculated at $y=y_{0}$. We now use the following property

$$
\frac{S_{0} y_{0}+T_{0}}{U_{0} y_{0}+V_{0}}=\frac{S_{0}+T_{0}}{U_{0}+V_{0}}=\frac{S_{0}}{U_{0}}=\frac{T_{0}}{V_{0}}=P_{0}
$$

to get

$$
D_{0}^{(13), s i n g .}=-\frac{\eta\left(i \epsilon_{1}, i \epsilon_{1}, \frac{i \epsilon_{1}}{y_{0}\left(y_{0}-1\right)}\right) \eta\left(i \epsilon \frac{P_{0}-1}{U_{0}+V_{0}},-i \epsilon \frac{P_{0}-1}{U_{0} y_{0}+V_{0}}, i \epsilon \frac{P_{0}-1}{P_{0}\left(U_{0}+V_{0}\right)}\right)}{\sqrt{\operatorname{det}(Q)}} .
$$

Thus, necessary and sufficient conditions for the function $D_{0}^{(13)}$ to have a LLS are

$$
\left\{\begin{array}{l}
\operatorname{det}(Q)=0 \\
0<y_{0}<1 \\
\left(U_{0}+V_{0}\right)\left(U_{0} y_{0}+V_{0}\right)<0 \text { and } T_{0} V_{0}<0
\end{array}\right.
$$

where $y_{0}$ is the root of the equation $S V-T U=0$. We have checked that this result agrees with the general conditions obtained by using Landau equations [6].

\section{B Basic integral and three-point Landau singularity}

We calculate the following integral

$$
\mathcal{S}_{1}=\int_{0}^{1} d y \frac{\ln \left(A y^{2}+B y+C-i \epsilon\right)}{y-y_{0}+i \epsilon^{\prime}},
$$


where $A$ is real, while $B, C$ and $y_{0}$ may be complex, with the restriction that the imaginary part of the argument of the logarithm has always the same sign for $0 \leq y \leq 1$, as indicated by the " $-i \epsilon "$. The " $-i \epsilon "$ is only needed when $y_{0}$ becomes real.

The numerator can be written as

$$
\ln \left(A y^{2}+B y+C-i \epsilon\right)=\ln (A-i \epsilon)+\ln \left(y-z_{1}\right)+\ln \left(y-z_{2}\right)+\eta\left(-z_{1},-z_{2}\right),
$$

where $z_{1,2}$ are the two roots of the argument of the logarithm. We have

$$
\mathcal{S}_{1}=\left[\ln (A-i \epsilon)+\eta\left(-z_{1},-z_{2}\right)\right] \ln \frac{y_{0}-1}{y_{0}}+\mathcal{R}_{1}\left(y_{0}, z_{1}\right)+\mathcal{R}_{1}\left(y_{0}, z_{2}\right),
$$

where the $\mathcal{R}_{1}$-function is defined as

$$
\mathcal{R}_{1}(y, z)=\int_{0}^{1} d x \frac{\ln \left(x-z+i \epsilon_{z}\right)}{x-y+i \epsilon_{y}}
$$

with $\epsilon_{z, y}$ are infinitesimal. If $y=z$ and being complex, we use

$$
\mathcal{R}_{1}(y, z)=\frac{1}{2} \ln \left(\frac{1-z}{-z}\right)[\ln (1-z)+\ln (-z)]
$$

If $y=z$ and being real, we then use

$$
\ln \left(x-z+i \epsilon_{z}\right)=\ln \left(x-z+i \epsilon_{y}\right)-\eta\left(x-z+i \epsilon_{z}, \frac{1}{x-z+i \epsilon_{y}}\right)
$$

to get

$$
\begin{aligned}
\mathcal{R}_{1}(y, z) & =\frac{1}{2} \ln \left(\frac{1-y}{-y}-i \epsilon_{y}\right)\left[\ln \left(1-y+i \epsilon_{y}\right)+\ln \left(-y+i \epsilon_{y}\right)+2 \eta\left(i \epsilon_{y},-i \epsilon_{z}, \frac{i \epsilon_{y}}{1-y}\right)\right] \\
& +\eta\left(i \epsilon_{y},-i \epsilon_{z}, \frac{i \epsilon_{y}}{y(y-1)}\right)\left[\ln \left(i \epsilon_{y}\right)-\ln \left(-y+i \epsilon_{y}\right)\right] .
\end{aligned}
$$

We observe that if $0 \leq y=z \leq 1$ and $\epsilon_{y} \epsilon_{z}<0$ then integration contour is pinched and this singularity corresponds to the anomalous threshold of scalar three-point integral (also called the three-point Landau singularity) whose nature is logarithmic. Since the value of the $\eta$-function is purely imaginary, the imaginary part of $\mathcal{R}_{1}$ is divergent while there is a finite jump in the real part of $\mathcal{R}_{1}$ at the singular point.

If $z \neq y$ then the result reads

$$
\mathcal{R}_{1}(y, z)=\ln (y-z) \ln \left(\frac{1-y}{-y}-i \epsilon_{y}\right)+\mathcal{R}(y, z)
$$

where the function $\mathcal{R}(y, z)$ is given in Appendix B of [7]. When calculating the rhs of Eq. (B.19), if the argument $(y-z)$ becomes negatively real then one has to introduce an infinitesimal imaginary part $i \epsilon_{y z}$. The result does not depend on the sign of this imaginary part as long as we introduce it everywhere in the rhs of Eq. (B.19). Thus, the simplest way is to write $y-z=y-z+i \epsilon_{z}$. This completes our calculation of $\mathcal{S}_{1}$. 


\section{References}

[1] L.D. Landau, Nucl. Phys. 13 (1959) 181.

[2] R. Eden et al., Cambridge University Press (1966).

[3] F. Boudjema and L.D. Ninh, Phys. Rev. D78 (2008) 093005, 0806.1498.

[4] S. Goria and G. Passarino, Nucl. Phys. Proc. Suppl. 183 (2008) 320, 0807.0698.

[5] S. Actis et al., Phys. Lett. B669 (2008) 62, 0809.1302.

[6] L.D. Ninh, PhD thesis, (2008), 0810.4078.

[7] G. 't Hooft and M.J.G. Veltman, Nucl. Phys. B153 (1979) 365.

[8] G.J. van Oldenborgh, Comput. Phys. Commun. 66 (1991) 1.

[9] G.J. van Oldenborgh and J.A.M. Vermaseren, Z. Phys. C46 (1990) 425.

[10] T. Hahn and M. Perez-Victoria, Comput. Phys. Commun. 118 (1999) 153, hep-ph/9807565.

[11] T. Hahn and M. Rauch, Nucl. Phys. Proc. Suppl. 157 (2006) 236, hep-ph/0601248.

[12] A. Denner, U. Nierste and R. Scharf, Nucl. Phys. B367 (1991) 637.

[13] S. Kawabata, Comp. Phys. Commun. 88 (1995) 309.

[14] D. Binosi and L. Theussl, Comput. Phys. Commun. 161 (2004) 76, hep-ph/0309015.

[15] S. Coleman and R.E. Norton, Nuovo Cim. 38 (1965) 438. 